\title{
Sleep quality of mother-caregivers of Duchenne muscular dystrophy patients
}

\author{
Karen T. Nozoe $^{1}$ • Daniel N. Polesel ${ }^{1}$ - Gustavo A. Moreira ${ }^{1} \cdot$ Gabriel N. Pires $^{1}$ • \\ Ricardo T. Akamine $^{1} \cdot$ Sergio Tufik ${ }^{1} \cdot$ Monica L. Andersen ${ }^{1}$
}

Received: 25 February 2015 /Revised: 7 May 2015 / Accepted: 11 May 2015 / Published online: 24 May 2015

(C) Springer-Verlag Berlin Heidelberg 2015

\begin{abstract}
Background Sleep disturbance is a common problem for caregivers. In general, patients with Duchenne muscular dystrophy (DMD) use noninvasive ventilation to maintain quality of life and improve survival.

Objective The aim of this study was to evaluate the sleep quality of caregiver-mothers of sons with DMD and factors that are associated with their sleep quality.

Methods We evaluated 32 caregiver-mothers of sons with DMD and 32 mothers of sons without any neuromuscular or chronic disease (control-CTRL group). The evaluation of quality of sleep was made using the Pittsburgh Sleep Quality Index (PSQI).

Results Caregiver-mothers had poor sleep quality, specifically longer sleep latency and reduced sleep efficiency. The impaired sleep quality of the caregiver-mothers was associated with the length of time of noninvasive ventilation used by their sons.

Conclusions Our results suggest that caregiver-mothers of sons with DMD have poor quality of sleep, and the length of use of noninvasive ventilation of their sons is associated with better sleep of caregiver-mothers.
\end{abstract}

Keywords Sleep · Caregiver · Bi-level positive airway pressure device (Bi-PAP) · Duchenne muscular dystrophy . Mothers $\cdot$ Noninvasive ventilation

Monica L. Andersen

ml.andersen12@gmail.com

1 Departamento de Psicobiologia, Universidade Federal de São Paulo, Rua Napoleão de Barros 925, 04024-002 São Paulo, SP, Brazil

\section{Introduction}

The importance of caregivers has increased substantially throughout the years. This is due to two main factors: an increase in the prevalence of some conditions that lead to the need for personal care $[1,2]$ and increased awareness regarding the importance of the role of caregivers in some conditions. However, such increased importance has highlighted the need for more attention to be paid towards the health and quality of life of the caregivers [3].

The role of a caregiver is associated with emotional, physical, social, and financial impairments [4], leading to particular stress conditions [5]. In general, caregivers tend to be women family members, usually a wife or mother. Caregiving has been linked to sleep disturbance $[6,7]$. Poor sleep quality is a common problem that affects caregivers of patients with chronic disease, especially in relation to shorter sleep times, decreased sleep efficiency, longer latency of sleep onset, more frequent awakenings after sleep onset, and daytime fatigue [7]. In caregivers of children with cancer, the caregivers have chronic partial sleep deprivation, poor sleep quality, and impaired daytime functioning [8,9]. Approximately two thirds of caregivers have sleep disorders [6]. These sleep disturbances lead to global health impairment, including mood and alertness disturbances [10] and sleepiness-related accidents [11].

Duchenne muscular dystrophy (DMD) is a neuromuscular disorder that causes serious health consequences that include a progressive inability to walk, impairment of the respiratory system [12], and sleep-disordered breathing [13]. Due to these conditions, the full-time presence of a caregiver is required in most cases.

Caregiver-mothers of patients with DMD are susceptible to sleep problems, and this, in turn, may affect their quality of life and health. However, the associated factors with this 
impairment of sleep are not well defined. Thus, our objective was to evaluate the subjective quality in caregiver-mothers of sons with DMD, as well as to identify associated factors with the poor quality of sleep of these caregiver-mothers.

\section{Material and methods}

\section{Study participants}

The group of caregiver-mothers of sons with DMD (caregivers) was recruited from the Center of Treatment of Neuromuscular Diseases, São Paulo, SP, Brazil. This center provides care for patients with neuromuscular disease, including DMD. The control (CTRL group, non-caregivers) was made up of mothers of sons without neuromuscular or chronic disease. The control subjects were recruited through disclosure and selected after interview if they met inclusion criteria. The subjects of the control group were matched in relation to age and body mass index (BMI) with the subjects of the caregiver-mother group. In both groups, the subjects were at least functionally literate. The inclusion criterion for the caregiver-mother group was to be a caregiver-mother of a son with DMD. For the CTRL group, the inclusion criterion was to be a mother of a son living at home without neuromuscular or chronic disease.

\section{Ethics statement}

This was a cross-sectional, observational study approved by the Ethical Committee of Research of the Federal University of São Paulo (CEP \#034176/2013) and is part of a larger study registered in ClinicalTrials.gov (Identification NCT01921374). All participants read and signed an informed consent form.

\section{Measures}

All volunteers were classified in relation to menstrual status, either as premenopausal or postmenopausal, as previously described in other studies $[14,15]$. BMI was calculated using the formula weight/height ${ }^{2}$.

Participants were questioned about the use of any drugs (e.g., antidepressants and oral contraceptives) and about previous illnesses, including depression. For caregiver-mothers of sons with DMD, information was collected about their sons, including age, diagnostic time, use of a wheelchair, age stopped walking, and use of noninvasive ventilation and the duration of use. This information was confirmed using the medical record of the son in our center. The diagnosis of DMD had been confirmed by muscle biopsy and/or genetic assessment.

\section{Questionnaire}

For sleep analysis, the Pittsburgh Sleep Quality Index (PSQI) was used. This is an instrument for evaluating perceived sleep quality during the previous month [16]. The questionnaire is composed of 19 questions subdivided into 7 components: subjective sleep quality, sleep latency, sleep duration, habitual sleep efficiency, sleep disturbances, use of a sleeping medication, and daytime dysfunction, which make up the final total score. Each component has a range scale of $0-3$, and the sum of the seven components make up the global PSQI score. Using the PSQI score, it is possible to define "good sleepers" (score $\leq 5$ ) and "poor sleepers" (score $>5$ ). This questionnaire has been validated in Portuguese language [17].

\section{Statistical analyses}

Data that did not meet the assumptions of normality and homogeneity were $z$-score-transformed for suitable parametricevaluation. The statistical analysis for the description of the sample group was carried out using the general linear model through one-way ANOVA. Chi-square test was used to determine the association between the categorical variables. To determine the possible associated factors with the caregiver-mother poor sleeper, a binary logistic regression was performed. The continuous variables chosen for the logistic regression were time of noninvasive ventilation (NIV) (months), age of son, and age and BMI of caregiver-mother. The categorical variable chosen for the logistic regression was use of a wheelchair by the son.

To obtain the value of the crude odds ratio, selected variables were analyzed one by one in the binary logistic regression using the Enter method. This evaluation enables identification of the odds ratio for each variable relative to the aim of the study. Sequence in the logistic test was conducted using the backward Wald method. This analysis first considers all selected variables and then removes them one by one until only those with the desired significance criterion remain. The significance level was set at $P \leq 0.05$. Data are reported as mean and standard deviation of the mean or frequency percentage.

\section{Results}

Our sample consisted of 32 caregiver-mothers and 32 control subjects, and the descriptive parameters show the characteristics of caregiver-mothers of children with DMD compared to mothers considered the CTRL group (Table 1).

The distribution of the sample according to scores of the questionnaire PSQI showed a higher frequency of poor sleep in the caregiver-mothers group $(61.3 \%)$ when compared to the control group $(29 \%)\left(\chi^{2}=6.513, d f=1, P=0.011\right)$. 
Table 1 Demographic data of the caregiver-mothers and control group

\begin{tabular}{llll}
\hline & Control group, $N=32$ & $\begin{array}{l}\text { Caregiver-mother } \\
\text { group, } N=32\end{array}$ & $P$ \\
\hline Age (years) & $46.0 \pm 7.1$ & $46.2 \pm 8.1$ & 0.870 \\
Body mass index & $26.7 \pm 3.9$ & $26.1 \pm 6.2$ & 0.632 \\
Number of children $(n)$ & $2.1 \pm 0.6$ & $2.3 \pm 1.0$ & 0.229 \\
Marital status (with partner) & $25(78.1 \%)$ & $24(75 \%)$ & 0.768 \\
Menopause (yes) & $13(40.6 \%)$ & $13(40.6 \%)$ & 1.000 \\
Practice of physical exercise (yes) & $9(36 \%)$ & $6(21.4 \%)$ & 0.240 \\
Use of an antidepressant (yes) & $1(3.1 \%)$ & $4(12.5 \%)$ & 0.162 \\
Ethnicity & $19(61.3 \%)$ & $18(56.2 \%)$ & 0.961 \\
$\quad$ White & $3(9.7 \%)$ & $3(9.4 \%)$ & \\
Afro-Brazilian & $6(19.4 \%)$ & $8(25 \%)$ & 0.294 \\
Native & $3(9.7 \%)$ & $3(9.4 \%)$ & \\
$\quad$ Asian or others/unknown & $12(38.7 \%)$ & $18(58.1 \%)$ & \\
Years of study & $10(32.3 \%)$ & $6(19.4 \%)$ & $7(22.6 \%)$ \\
$\quad$ Up to 8 years & $9(29 \%)$ & & \\
Up to 11 years & & & \\
Up to 15 years &
\end{tabular}

Table 2 presents the frequency and mean of the demographic data of the sons of caregiver-mothers with good sleep and poor sleep. The following factors were not significant between the two groups: age of son $\left(F_{1,26}=1.992\right.$; $P=0.192)$, time since diagnosis in months $\left(F_{1,26}=1.062\right.$; $P=0.312)$, wheelchair use $\left(\chi^{2}=89, d f=1, P=0.765\right)$, and use of NIV $\left(\chi^{2}=1.094, d f=1, P=0.296\right)$. The percentage of NIV use $\left(F_{1,16}=506 ; P=0.487\right)$ and mean of daily NIV use $\left(F_{1,16}=875 ; P=0.363\right)$ were also found to be not statistically different between groups. However, having been using NIV over a short period appears to have an effect on the sleep of caregiver-mothers. The sons of mothers who were classified as good sleepers had an average of 63 months use of the NIV device, while the sons of poor sleepers had only 24 months of mean of use $\left(F_{1,26}=5.101\right.$; $P=0.033)$. The length of time in months of use of the NIV device by son of caregiver-mothers, poor and good sleeper, is depicted in Fig. 1.
Table 4 depicts the logistic model used to identify the associated factors for poor sleep in caregivermothers. The length of time of use of noninvasive ventilation shows that the model has statistical reliability $\left(-2 \mathrm{LL}=33.535\right.$; Cox-Snell $R^{2}=0.155$; Nagelkerke $R^{2}=$ 0.208). The length of the time of use of noninvasive ventilation was considered a protective factor related to good sleepers (adjusted OR $=0.98$, CI $95 \%$ 0.96-0.99; $P=0.024)$.

\section{Discussion}

In the present study, over $60 \%$ of the caregiver-mothers had impairment of their sleep, particularly longer sleep latency. The short time of use of the NIV device by their sons was the factor associated with the poor sleep.
Table 2 Demographic data of the sons of caregiver-mothers

\begin{tabular}{llllr}
\hline & $\begin{array}{l}\text { All caregiver-mothers, } \\
N=31\end{array}$ & $\begin{array}{l}\text { Caregiver-mother } \\
\text { poor sleeper, } N=12\end{array}$ & $\begin{array}{l}\text { Caregiver-mother } \\
\text { good sleeper, } N=19\end{array}$ & $P$ \\
\hline Age of son (years) & $18.7 \pm 6.0$ & $17.3 \pm 5.9$ & $20.3 \pm 5.8$ & 0.192 \\
Time since diagnosis (months) & $158.6 \pm 73.0$ & $145.1 \pm 66.4$ & $172.0 \pm 70.8$ & 0.312 \\
Son is a wheelchair user (yes) & $28(93.3 \%)$ & $17(94.4 \%)$ & $11(91.7 \%)$ & 0.765 \\
Regular use of NIV (yes) & $16(55.2 \%)$ & $8(66.7 \%)$ & $8(47.1 \%)$ & 0.296 \\
Nights used (\%) & $97.4 \pm 6.2$ & $98.3 \pm 3.4$ & $96.2 \pm 8.7$ & 0.487 \\
Mean of daily use (h) & $11.3 \pm 4.1$ & $12.3 \pm 8.7$ & $10.1 \pm 9.4$ & 0.363 \\
Duration of NIV treatment (months) & $41.2 \pm 45.7$ & $24.8 \pm 36.3$ & $63.0 \pm 50.4 *$ & 0.033 \\
\hline
\end{tabular}

$N I V$ noninvasive ventilation

*P statistical significance 


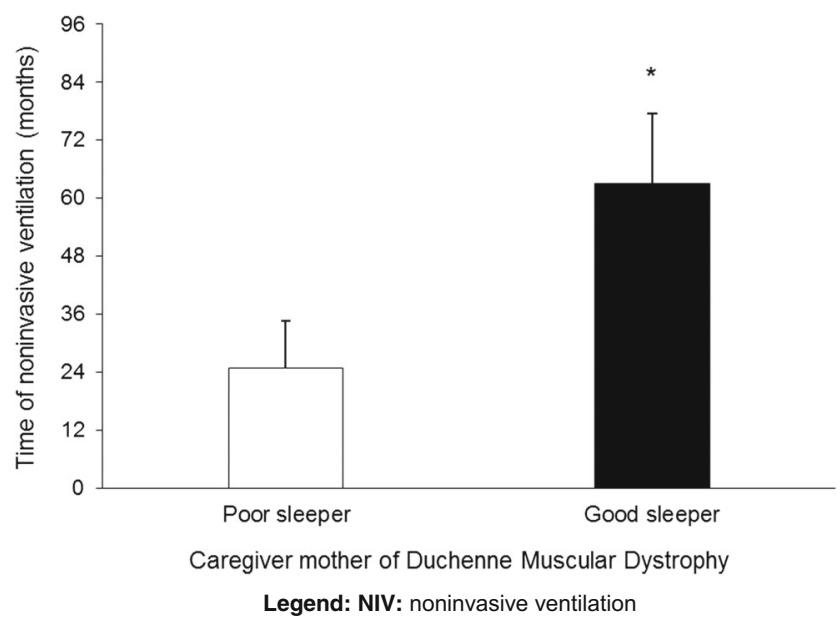

Fig. 1 Duration of noninvasive ventilation treatment of the caregivermother group for those with poor sleep and good sleep. NIV noninvasive ventilation. $* P<0.05$ compared with the poor sleeper group

Studies have demonstrated that caregivers have poorer sleep than non-caregivers $[18,19]$. In our study, we also observed that caregiver-mothers have worse sleep quality than non-caregivers, especially in the domain of sleep latency. When observing the group of mother-caregivers we noted that the group is mainly affected in aspects of subjective sleep quality, sleep latency, sleep disturbances, and overall quality of sleep (Table 3). In relation to caregivers of children who used NIV, impairment in sleep caused by having to turn on the equipment or to turn off the alarm has been observed [20,21]. In our sample of caregiver-mothers, $55.2 \%$ had sons who used NIV. Interestingly, duration of NIV treatment was associated with poor sleep of caregiver-mothers (Table 4). As shown in Fig. 1, the length of time of use of NIV was inversely related to the quality of sleep of the mother. Thus, the caregiver-mothers who had a poor quality of sleep were those whose sons had been using the NIV for a relatively shorter time (average of approximately 25 months). The caregivermothers who had good sleep quality were the mothers of sons who had been using NIV for a long time (about 63 months). We emphasize the relevance of this finding of our study, since the percentage of use of NIV and the average daily time of use were not significantly different between the poor sleeper and good sleeper groups of caregiver-mothers. The fact that the length of use of NIV has this effect may be related to the better psychological adjustment of the mother and/or behavior and sleep of their sons. Patients with DMD have impaired sleep patterns, especially sleep-disordered breathing, obstructive sleep apnea [22], and hypoventilation [23]. Other sleep disorders include difficulty in initiating and maintaining sleep. Many patients show significant sleep fragmentation with frequent awakenings for changes in body positions (by the caregivers) due to pain or discomfort [24]. For these reasons, NIV is indicated for DMD patients, a therapy that provides relative "rest" for the respiratory muscles, improves the ventilatory response to hypercapnia, and improves respiratory performance (reduction in thoracoabdominal asynchrony and recruiting areas of atelectasis). Thus, sleep patterns are represented by the decrease in respiratory events, sleep fragmentation, increased total sleep time, and REM sleep periods [25, 26]. The use of NIV also increases the life expectancy of these patients [27]. However, patients depend on the help of a caregiver to turn on and adjust the equipment, especially when the patient is a child or has DMD which restricts movement.

NIV is associated with some risks, such as malfunction or disconnection of equipment or power loss which can cause the death of the patient who is dependent on NIV $[28,29]$. For this reason, the period at the beginning of use of the equipment can be a time of adaptation for both the patient and the caregiver-mother. Therefore, the explanation to our findings might lie in the fact that after the period of adaptation to NIV, the patient presents a continuous sleep without fragmentation and that this is consequently reflected in the improved quality of sleep of the caregiver-mother.

The mean age of sons of caregiver-mothers was $18.7 \pm$ 5.9 years, while caregiver-mothers with good sleep quality had sons with a mean age of $20.3 \pm 5.8$ years and caregivermothers with a poorer quality of sleep had sons with a mean age of $17.3 \pm 5.9$ years.
Table 3 Components of the Pittsburgh Sleep Quality Index

\begin{tabular}{llll}
\hline Components (domain) & Control group, $N=31$ & Caregiver-mothers, $N=31$ & $P$ \\
\hline Subjective sleep quality (D1) & $0.65 \pm 0.66$ & $1.70 \pm 1.06$ & 0.128 \\
Sleep latency (D2) & $0.74 \pm 0.63$ & $1.33 \pm 1.13^{\mathrm{a}}$ & 0.014 \\
Sleep duration (D3) & $1.10 \pm 0.87$ & $1.23 \pm 0.88$ & 0.549 \\
Habitual sleep efficiency (D4) & $0.45 \pm 0.92$ & $1.00 \pm 1.18^{\mathrm{a}}$ & 0.050 \\
Sleep disturbances (D5) & $1.32 \pm 0.54$ & $1.40 \pm 0.50$ & 0.563 \\
Use of a sleeping medication (D6) & $0.10 \pm 0.40$ & $0.40 \pm 0.92$ & 0.102 \\
Daytime dysfunction (D7) & $0.26 \pm 0.58$ & $0.40 \pm 0.72$ & 0.399 \\
Total score & $4.61 \pm 3.9$ & $7.17 \pm 2.3^{\mathrm{a}}$ & 0.003 \\
\hline
\end{tabular}

a $* P<0.05$ compared with the control group 
Table 4 Binary logistic regression model for calculation of the adjusted odds ratio $(\mathrm{aOR})$ related to the poor sleeper group of caregiver-mothers

Factors associated with poor sleep of caregiver-mothers

\begin{tabular}{lllllll}
\hline & COR & $\begin{array}{l}95 \% \mathrm{CI} \\
\text { for COR }\end{array}$ & $P$ & AOR & $\begin{array}{l}\text { 95\% CI } \\
\text { for AOR }\end{array}$ & $P$ \\
\hline Constant & - & - & - & 3.708 & - & 0.020 \\
Duration of NIV treatment (months) & 0.98 & $0.96-0.99$ & 0.024 & 0.98 & $0.96-0.99$ & 0.024 \\
Son is a wheelchair user (yes) & 0.65 & $0.04-11.45$ & 0.767 & - & - & - \\
Age of son & 0.92 & $0.81-1.04$ & 0.189 & - & - & - \\
Age of caregiver-mother & 0.90 & $0.80-1.01$ & 0.060 & - & - & - \\
BMI of caregiver-mother & 1.09 & $0.92-1.30$ & 0.331 & - & - & - \\
\hline
\end{tabular}

$A O R$ adjusted odds ratio, $N I V$ noninvasive ventilation, $B M I$ body mass index, $C I$ confidence interval, $C O R$ crude odds ratio

The effects in mothers of children with chronic illness is usually greatest at the time of diagnosis but decreases with subsequent adaptation due to better acceptance and the adoption of appropriate coping strategies [30, 31], suggesting that the coping and adjustment are more relevant to the psychological outcome than the disease severity [32]. Psychological stress has an effect on sleep pattern. Sleep impairment is associated with health problems, such as cognitive deficits [33,34] and sleepiness-related accidents [11]. Thus, we would like to emphasize the importance of good sleep quality for the caregiver-mother, since this may compromise the activities and duties of the caregiver as well as the patient care that is crucial for her son's survival and quality of life. Furthermore, another study suggests that poor caregiver sleep decreases the quality of life and increases the risk of morbidity [35]. Caregiver-mothers, as well as their sons with DMD, may benefit from an evaluation in the home by a medical team.

Some limitations of the study need to be considered. First, the study has a small sample size, an issue that may affect the accuracy and generalizability of the results. Second, sleep quality of the sons was not evaluated, as we prioritized the caregiver-mothers. Third, our study was based on a subjective analysis of sleep and an objective analysis may complement the subjective findings. Fourth, we did not perform a longitudinal evaluation of sleep quality before and after NIV.

\section{Conclusion}

Our results suggest that quality of sleep is poor in caregivermothers of sons with DMD, especially those whose son has only used noninvasive ventilation for a short length of time. The longer-term use of NIV appears to result in an adaptation by the caregiver-mother with consequent improvement in sleep quality. Lastly, these results demonstrate the importance and necessity of research on action to facilitate the use of NIV in patients with DMD. The initial months of beginning NIV is a critical time for caregivers during which they need intensive social support. The findings of our study emphasize the importance of achieving good sleep and good health for the caregiver who is responsible for providing support to the patient.

Acknowledgments This work was supported by grants from Associação Fundo de Incentivo a Pesquisa (AFIP), CAPES, CNPq (M.L.A. and S.T. are recipients of the CNPq fellowship), and São Paulo Research Foundation (FAPESP) (\#2014/08067-0 to KTN).

Conflict of interest None of the authors have any conflict of interest to disclose. We confirm that we have read the journal's position on issues involved in ethical publication and affirm that this report is consistent with those guidelines.

\section{References}

1. Peña-Longobardo LM, Oliva-Moreno J (2015) Caregiver burden in Alzheimer's disease patients in Spain. J Alzheimers Dis 43:1293302

2. Sautter JM, Tulsky JA, Johnson KS, Olsen MK, Burton-Chase AM, Lindquist JH et al (2014) Caregiver experience during advanced chronic illness and last year of life. J Am Geriatr Soc 62:1082-90

3. Kim Y, Schulz R, Carver CS (2007) Benefit-finding in the cancer caregiving experience. Psychosom Med 69:283-91

4. Connell T, Griffiths R, Fernandez RS, Tran D, Agar M, Harlum J (2011) Quality-of-life trajectory of clients and carers referred to a community palliative care service. Int J Palliat Nurs 17:80-5

5. Bevans M, Sternberg EM (2012) Caregiving burden, stress, and health effects among family caregivers of adult cancer patients. JAMA 307:398-403

6. Kim H, Rose K (2011) Sleep disturbances in family caregivers: an overview of the state of the science. Arch Psychiatr Nurs 25:456-68

7. Pawl JD, Lee SY, Clark PC, Sherwood PR (2013) Sleep loss and its effects on health of family caregivers of individuals with primary malignant brain tumors. Res Nurs Health 36:386-99

8. Meltzer LJ, Mindell JA (2006) Impact of a child's chronic illness on maternal sleep and daytime functioning. Arch Intern Med 166: 1749-55

9. Boman K, Lindahl A, Björk O (2003) Disease-related distress in parents of children with cancer at various stages after the time of diagnosis. Acta Oncol 42:137-46 
10. Wehrens SM, Hampton SM, Kerkhofs M, Skene DJ (2012) Mood, alertness, and performance in response to sleep deprivation and recovery sleep in experienced shiftworkers versus nonshiftworkers. Chronobiol Int 29:537-48

11. Pandi-Perumal SR, Verster JC, Kayumov L, Lowe AD, Santana MG, Pires ML et al (2006) Sleep disorders, sleepiness and traffic safety: a public health menace. Braz J Med Biol Res 39:863-71

12. Sarnat HB (2000) Neuromuscular disorders. Nelson textbook of pediatrics. WB Saunders, Philadelphia, pp 1881-2

13. Barbé F, Quera-Salva MA, McCann C, Gajdos P, Raphael JC, de Lattre J et al (1994) Sleep-related respiratory disturbances in patients with Duchenne muscular dystrophy. Eur Respir J 7:1403-8

14. Hachul H, Andersen ML, Bittencourt L, Santos-Silva R, Tufik S (2013) A population-based survey on the influence of the menstrual cycle and the use of hormonal contraceptives on sleep patterns in São Paulo, Brazil. Int J Gynaecol Obstet 120:137-40

15. Nozoe KT, Hachul H, Hirotsu C, Polesel DN, Moreira GA, Tufik S et al (2014) The relationship between sexual function and quality of sleep in caregiving mothers of sons with Duchenne muscular dystrophy. Sex Med 2:133-40

16. Buysse DJ, Reynolds CF, Monk TH, Berman SR, Kupfer DJ (1989) The Pittsburgh Sleep Quality Index: a new instrument for psychiatric practice and research. Psychiatry Res 28:193-213

17. Bertolazi AN, Fagondes SC, Hoff LS, Dartora EG, Miozzo IC, de Barba ME et al (2011) Validation of the Brazilian Portuguese version of the Pittsburgh sleep quality index. Sleep Med 12:70-5

18. Brummett BH, Babyak MA, Siegler IC, Vitaliano PP, Ballard EL, Gwyther LP et al (2006) Associations among perceptions of social support, negative affect, and quality of sleep in caregivers and noncaregivers. Health Psychol 25:220-5

19. McCurry SM, Logsdon RG, Teri L, Vitiello MV (2007) Sleep disturbances in caregivers of persons with dementia: contributing factors and treatment implications. Sleep Med Rev 11:143-53

20. Andrews MM, Nielson DW (1988) Technology dependent children in the home. Pediatr Nurs 14(111-4):51

21. Wang KW, Barnard A (2004) Technology-dependent children and their families: a review. J Adv Nurs 45:36-46

22. Nozoe KT, Moreira GA, Tolino JR, Pradella-Hallinan M, Tufik S, Andersen ML (2015) The sleep characteristics in symptomatic patients with Duchenne muscular dystrophy. Sleep Breath. doi:10. 1007/s11325-014-1103-9

23. Suresh S, Wales P, Dakin C, Harris MA, Cooper DG (2005) Sleeprelated breathing disorder in Duchenne muscular dystrophy: disease spectrum in the paediatric population. J Paediatr Child Health 41: $500-3$

24. Bloetzer C, Jeannet PY, Lynch B, Newman CJ (2012) Sleep disorders in boys with Duchenne muscular dystrophy. Acta Paediatr 101: 1265-9

25. Petrone A, Pavone M, Testa MB, Petreschi F, Bertini E, Cutrera R (2007) Noninvasive ventilation in children with spinal muscular atrophy types 1 and 2. Am J Phys Med Rehabil 86:216-21

26. Lisboa C, Díaz O, Fadic R (2003) Noninvasive mechanical ventilation in patients with neuromuscular diseases and in patients with chest restriction. Arch Bronconeumol 39:314-20

27. Vianello A, Bevilacqua M, Salvador V, Cardaioli C, Vincenti E (1994) Long-term nasal intermittent positive pressure ventilation in advanced Duchenne's muscular dystrophy. Chest 105:445-8

28. Nelson VS, Carroll JC, Hurvitz EA, Dean JM (1996) Home mechanical ventilation of children. Dev Med Child Neurol 38:704-15

29. Chestnut MA (1998) Pediatric home care manual: LippincottRaven

30. Folkman S, Lazarus RS, Gruen RJ, DeLongis A (1986) Appraisal, coping, health status, and psychological symptoms. J Pers Soc Psychol 50:571-9

31. Sheeran T, Marvin RS, Pianta RC (1997) Mothers' resolution of their child's diagnosis and self-reported measures of parenting stress, marital relations, and social support. J Pediatr Psychol 22: $197-212$

32. Nereo NE, Fee RJ, Hinton VJ (2003) Parental stress in mothers of boys with Duchenne muscular dystrophy. J Pediatr Psychol 28: 473-84

33. Alhola P, Polo-Kantola P (2007) Sleep deprivation: impact on cognitive performance. Neuropsychiatr Dis Treat 3:553-67

34. Durmer JS, Dinges DF (2005) Neurocognitive consequences of sleep deprivation. Semin Neurol 25:117-29

35. Schulz R, Martire LM (2004) Family caregiving of persons with dementia: prevalence, health effects, and support strategies. Am J Geriatr Psychiatry 12:240-9 